\title{
Beyond Intuition: Towards a Framework for Empirical-Based Design Theory Building in Design Science Research
}

\author{
Marius Mueller, Oliver Heger, Bastian Kordyaka, Henrik Kampling, Bjoern Niehaves \\ University of Siegen \\ \{marius.mueller | oliver.heger | bastian.kordyaka | henrik.kampling | bjoern.hiehaves\}@ uni-siegen.de
}

\begin{abstract}
Over the past twenty years, Design Science Research (DSR) has received major attention within the IS domain. Besides constructing and evaluating artefacts, researchers put effort into theorizing on IT design and its effects on users. Here, the development and testing of design theories is of major interest. Yet, design theory studies often lack empirical investigations on the identification of appropriate design features. Whereas in general DSR activities incorporate empirical investigations on many levels, the intertwined development of a theoretical model in connection with design features can further profit from empirical investigations by exploring the design realm of a specific context. We therefore propose a qualitative five-step approach suitable for inducing design features and theoretical constructs by engaging experienced stakeholders. We present a case study on the development of a support system for physiotherapeutic treatments, illustrating the proposed approach.
\end{abstract}

\section{Introduction}

Throughout the last two decades, Design Science Research (DSR) has experienced a major ascent, drawing more and more attention of Information Systems (IS) researchers to the scientific exploration, testing, and validation of IT artefact design. Looking at the history of DSR and the accompanying research agendas, practical and theoretical DSR activities have been treated rather dichotomously. Nevertheless, researchers increasingly conclude that theory and practice are thoroughly interwoven and can inform each other [25]. Thus, the construction, implementation, and evaluation of new IT artefacts were complemented by studies establishing theoretical foundations for proper technology design. As a result, the term "design theory" (DT) was popularized, though the researchers' conceptions of a DT have been diverse (e.g. $[2,12])$. DTs are highly valuable for DSR since they explain how and why specific design features have desired or undesired effects.
The understanding of what constitutes a design theory underwent an evolutionary development. Most recently, the concept of an explanatory Information System Design Theory (ISDT) has been proposed. In the vein of artefact construction, evaluation, and theorizing, an ISDT seeks to integrate technical and theoretical considerations, including a normative reasoning of effects hypothesized causes may have [25]. Through systematic manipulation of design features, which operationalize one or more independent variables (i.e. causes of effects), a proposed design theory can be tested within experimental and controlled set-ups [40].

Not only the understanding, but also the approach for developing a design theory is still evolving. When it comes to building a design theory model, which includes the development of design features for specific purposes, researchers, in most cases, make use of external theoretical insights in connection with their intuition for design (e.g. [38]). Hence, a lack of empirical foundation of the design process becomes evident. Besides intuition and/or de-contextualized factors as source of inspiration, appropriate technology design is rooted in context-bound, empirical evidence, for instance, generated by problem solving [13]. Due to the specific contexts new IT artefacts operate in, the transfer of external design knowledge in combination with the researcher's intuition might fall short in addressing the demands, technical and social requirements, and user concerns associated with the prevailing context. Although empirical investigations are applied for design theories by the experimental testing of a proposed theory, the operationalization of manipulable, independent variables in the shape of varying design features are often insufficiently founded in empirical investigations. Due to the situative nature of an IT artefact within an application area, researchers have difficulties to anticipate the cause-effect relations between specific design features and theoretical constructs. To be clear, we do not claim that DSR lacks inductive approaches in general. We specifically refer to the development of appropriate design features within design theory development.

To this end, empirical-based reasoning and inquiry can help to develop design theories with regard to 
different quality criteria such as feasibility, usefulness, and desirability of the technology design. When it comes to conducting empirical investigations boasting indefinite goals, courses, and results, explorative, qualitative research methods have proven to address these issues adequately (see for instance [39]). In this regard, focus group research (FGR) resembles an economically advantageous approach able to produce rich and explorative data $[26,33]$ and, thus, exhibits a high degree of suitability to inform the development of ISDTs. In this paper, we propose an empirical method aiming at the formation of context-bound, highly relevant design principles and associated theoretical constructs, enabling the empirically grounded development of design theories. Due to the duality of artefact and theory testing in our method, the specific design of an artefact is validated by validating the design theory, while the design theory is validated by validating the artefact.

\section{Theoretical Background}

\subsection{Design Science and Theory Building}

Design Science Research (DSR) resembles a major research field within the Information Systems (IS) discipline and has been driving research agendas for more than 20 years [25]. In this regard, many theoretical and practical basics have been developed throughout the years such as guidelines for the proper execution and evaluation of DSR activities [14], methodological frameworks dividing the underlying research processes into distinct steps (i.e. problem identification, goals, design, and evaluation) and illustrating them within use cases [28].

Theory building represents a major component of modern DSR. The discipline, besides creating and evaluating IT artefacts, strives to gain theoretical insights and build theoretical foundations. However, design theorizing within IS research has been critically discussed. According to March and Smith, for instance, theory building is rooted in behavioral science, hence it is not suitable to inform design-oriented disciplines with a technological focus such as IS [21]. In contrast, IT artefacts are implemented and used within social contexts, where human behavior is of major interest with regard to technology design. Hence, analyzing technologies under development through a theoretical lens is able to deliver valuable insights on IT construction and its impacts [25].

The term "design theory" (DT) has been extensively dealt with in scientific literature. Baskerville and Pries-Heje, for instance, define different characteristics a DT should exhibit such as its prescriptive nature and its practical application to inform future design choices and actions [2]. Further, a DT can be seen as a dual concept, covering a product and a process dimension. The product dimension refers to the DT's form and function, whereas the process dimension addresses the scientifically substantiated development process of an IT artefact [12, 37]. This duality leads to the definition of two kinds of DT, which have been treated dichotomously in the past. (1) Design practice theories (DPT) specify how to construct an artefact, referring to the process dimension. (2) Explanatory design theories (EDT), on the contrary, specify why to integrate a specific feature within an IT artefact [2]. With regard to EDT, the "exterior mode" of a DT seeks to answer the question on why an artefact with a specific design feature delivers better results than another artefact, which do not have this feature [11]. Similarly, a design-relevant explanatory/predictive theory (DREPT) seeks to investigate effects an artefact causes [19].

All of the above imply a conceptual separation of artefact construction and theory, thus, "evaluation results cannot be traced back to particular features" [25] (p. 2). This detachment of theory from IT development can be counteracted by applying so-called "kernel theories", which form a knowledge base for DT construction and allow to draw on adjacent disciplines such as social sciences and psychology. This lead to the definition of an explanatory IS design theory (ISDT), consisting of "two or more connected hypotheses" (ibd. p. 4), each hypothesis describing a cause-effect-relation between independent (IV) and dependent variables (DV). Hereby, at least one IV needs to be systematically manipulable through design, additionally "at least one dependent variable is regarded as desirable or undesirable" (ibd. p. 4).

According to this conceptual understanding and definition of a design theory, theorizing within the DSR domain requires specific and technically implementable design features to which effects and theoretical insights can be traced back. The choice of design variations operationalizing the independent variable(s) of the kernel theory is by far no trivial task and is often rooted in intuition, expertise, or, in other words, abductive approaches towards occurring effects. For some researchers, abduction can offer valuable contributions for eliciting intelligent solutions [6]. Abduction in design science is described as having a putative role in scientific inquiry and knowledge creation [3].

However, this being said, ISDT construction particularly lacks the empirical foundation of design items, which in many cases are embedded within specific, technological research and development contexts and, thus, exhibit a situative character. This calls for a closely intertwined perspective on theory 
and artefact design and especially novel ways of design item development.

\subsection{Quality Criteria of Design Choices}

Throughout literature, several indicators can be found that address the quality of an IT artefact and the design features it incorporates. In the context of DSR and design theory building, examinations with regard to three major quality criteria appear reasonable: (1) feasibility of the IT artefact design (i.e. usefulness and added value), (2) ethical considerations (i.e. desirability and acceptability), and (3) its suitability for scientific investigations within a given context (i.e. it evokes significant effects that can be measured).

Feasibility studies represent a widely used tool to assess whether an IT artefact's development is reasonable from different perspectives (e.g. [29]). Low degrees of feasibility are fostered by the absence of usefulness, alleviating the acceptance of the artefact once it is brought to use [36]. In their study on evaluating the feasibility of information systems within healthcare und telemedicine, Judi et al. identify four aspects that contribute to technological feasibility, i.e. operational, time, economic, and technical aspects [15]. Operational aspects relate to the way the IT artefact fulfils operational needs and can be implemented in pre-existing structures and workflows. The time factor addresses the amount of resources the artefact requires to be properly used. From an economic perspective, (initial and ongoing) financial investments relate to long-term benefits, which are able to antagonize inhibitions regarding the purchase of the IT artefact. The technical aspect covers investigations on whether the artefact fulfils requirements the given infrastructure imposes, enabling a proper implementation. When it comes to building an ISDT, thus, the feasibility of the design items needs to be assessed beforehand.

Ethical considerations with regard to technology design choices and the impacts the information system will have (e.g. on society, individuals, and institutions) especially concern the desirability and acceptability of an IT artefact. In order to assess these aspects of the IT design, exploring the value systems of relevant stakeholders (developers, users, peers etc.) can help to identify what is desirable. In this regard, while closely linked to the ethical and philosophical realm, the Value Sensitive Design approach [9] provides ways to unveil important values and to transfer them into concrete technological features. The integration of design features, which do not hold high levels of acceptability and desirability, appears to have no practical meaning within an ISDT.
The third quality criteria presented in this paper is formed by the degree of scientific relevance and suitability. An ISDT involves one or more hypotheses, which represent cause-effect relations. In this regard, those design alterations (i.e. design items) appear suitable and theoretically profitable, that promise significant effects. In literature, three dominant ways of identifying design alternatives for the sake of theory building can be detected: (1) intuition and/or experience, (2) derivation from other studies and adaptation, and (3) a mixture of both (see for instance [40]). As mentioned above, design choices are contextbound in most cases, evoking certain effects within specific situations and under specific circumstances. Hence, the adaptation to another context (i.e. a new technological realm with differing use behavior and associated effects) might be inadequate, leading to unintended effects. Therefore, a researcher cannot expect that adapted design items lead to intended (or anticipated) effects. This calls for a more empiricalbased foundation.

As a prerequisite of IT design within a certain context (e.g. an enterprise) addressing specific tasks (e.g. decision making), technological expertise and tacit knowledge form the foundation of design-relevant choices. In order to address the quality criteria above, the empirical involvement of context-aware stakeholders, i.e. experts regarding artefact development and its application, appears to be necessary.

\section{Method Development}

\subsection{Explorative Approach}

Within DSR, the construction and testing of design theories often takes places in connection with novel technological advancements, where the appropriate design choices have not been developed yet. By recognizing this duality of design theory and artefact design, an ISDT, thus, is able to deliver rich insights into the effects the application of an IT artefact will have and how these effects are connected to concrete design features. Here, the utilization of qualitative methods is able to inform the construction of an ISDT based on empirical evidence. Without intending to ignore the value of abduction in design items development, we propose an empirical approach since we argue that ISDT construction particularly lacks the empirical foundation of design items.

In this regard, the focus group research (FGR) approach represents a promising method for a guided and focused exploration while engaging a group of chosen individuals (i.e. relevant stakeholders) from diverse disciplines, each with different inherent values, 
meanings and attitudes [30, 33]. This approach represents a method for empirical data collection "through group interaction on a topic determined by the researcher" [24]. The topical focus is generated by the goals the participants have in common, for instance, the design of an IT artefact within a joint research project. A focus group session is guided by one or more moderators, who initiate a mutual discussion among the participants through open-ended and narratively stimulating questions [26, 34].

Up to date, the utilization of focus groups as a data generating method is highly underutilized in the Information Systems domain [26, 33]. However, FGR exhibits several strengths, from which DSR researchers and design theorizing can benefit: (1) Group interaction and arising discussion allow for collective reasoning and value generation through mutual negotiation, enabling the emergence of consensus as well as conflicts [26, 30, 33]. (2) FGR can increase the depth of data through surfacing opinions and ideas that would otherwise not be externalized, thus filling individual knowledge gaps and creating a shared space of understanding [8, 33]. (3) The inclusion of minorities within a FGR approach can increase the participants' self-esteem and willingness to take part in the discussion [17]. (4) Utilizing a FGR approach comes with notable economic benefits regarding the time and resources researchers have to invest. In comparison to other methods (e.g. interviews), focus groups are able to produce large amounts of valuable data within short time frames [33]. Before conducting empirical investigations in order to explore promising design features and integrate them into a new ISDT, several prerequisites need to be fulfilled.

Firstly, the purpose and scope of the technological artefact under construction need to be clear. When it comes to designing and testing an IT artefact and its components, researchers and designers operate within a specific context regarding technological (e.g. existing infrastructure) as well as social factors (e.g. use behavior and attitudes towards the artefact) following superordinate goals. Hence, the boundaries of the artefact and the associated ISDT need to be explored and defined. In this regard, the definition of usage scenarios offers theoretical and practical clarity [5]. Scenario building is able to deliver a comprehensive understanding of occurring forms of applications, different stakeholders and user groups, technological and social requirements, as well as conflicts that might arise when it comes to implementing the IT artefact within the targeted context. The latter is particularly important with regard to the ethical aspects of technology design, as these address many possible outcomes, creating a normative reasoning in ISDT building [27]. Secondly and closely linked to scenario building, the acquisition of relevant stakeholders and expertise enables researchers to build normative inquiry based on diverse value systems, experience and (tacit) knowledge in a deliberative manner [27]. From an empirical perspective, by involving relevant experts during the theory construction phase, researchers have access to fundamental knowledge with regard to artefact design and requirements from context-bound perspectives. For instance, a medical practitioner can judge on proposed technology specifications of a system intended to support therapeutic activities from a more context-related perspective (e.g. [1]).

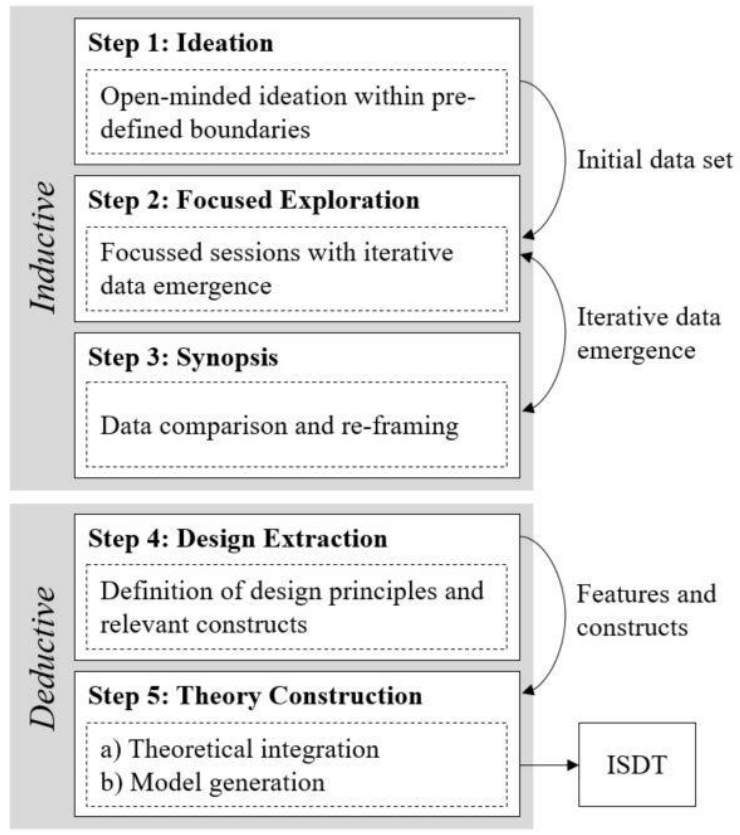

Figure 1. Theory development procedure.

\subsection{Data-driven Design Theory Building}

The empirical data generation procedure presented in this paper follows an explorative focus group approach and is presented in Figure 1. The procedure consists of five consecutive steps, involving an inductive (steps 1 to 3 ) and a deductive phase (steps 4 and 5). The inductive steps serve the purpose of initial, followed by iterative data collection and coding. Subsequently, the deductive steps seek to integrate the gathered data and derived insights within adjacent literature in order to identify valuable kernel theories and relating constructs, followed by the final construction of an ISDT model. Subsequently, the empirical ISDT construction procedure is described. Since design science is an iterative process, the procedure proposed here represents one iteration and merely a part of an overall complex design study.

Step 1: Ideation. Within the first methodological step, an open-minded ideation session engaging 
relevant stakeholders from research and practice takes place in order to unveil an initial set of design requirements and associated expectations, issues, societal impacts etc. Here, a "full group" approach acquiring multiple perspectives and diverse knowledge [10] appears to deliver rich insights, informing further investigations and discussions. The baseline for discussion and discourse initiation is formed by the collection and definition of usage scenarios. In many cases, these scenarios are partly pre-defined due to the predominant project boundaries and technological purpose. Due to the involvement of a broad spectrum of disciplines, each incorporating its own priorities, opinions, and meanings, a rigor moderation is necessary to evoke fruitful discussions and suppress unwanted group dynamics and conflicts [26, 32]. The heterogeneous participant constellation al-lows for interdisciplinary discussion and interaction, stimulating "out-of-the-box" thinking and mutual perspective taking. Uprising concerns regarding potential hazardous design choices (both from a technological and a use-related standpoint) can be dissolved on the spot, enriching the design's feasibility and acceptability. Following the focus group session, the gathered (transcribed) data is initially coded. This resembles the coding procedure utilized within the Grounded Theory approach [35, 39] and aims at forming a first categorization scheme covering emerged themes.

Step 2: Focused Exploration. Following the initial data collection step, the focused exploration encompasses targeted investigations from different perspectives. Here, the themes identified in step 1 form the baseline for further focus group sessions. In order to dive deeper into the respective themes and to reach a high degree of comparability across the involved disciplines, the focused exploration stipulates the conduction of multiple sessions, each involving only stakeholders from one specific domain, following a purposeful sampling procedure [22]. The number of necessary workshops aligns with the quantity of involved parties. These homogeneous groups serve the purpose of illuminating each identified superordinate theme from one specialized point of view, delivering distinct sets of data. Hereby, each session consists of an initial, open-ended phase of a rather narrative nature, discussing each theme impartially. Subsequently, the moderator uses statements from a previous session as a stimulating input for further discussion. This allows for a constant comparison between each session and, thus, stakeholder group, as well as theoretically driven, iterative data emergence. Conflicts, which are identified by the moderator during a session and relate to contradicting statements and opinions, can be addressed on the spot. In this regard, the moderator(s) should be aware of potentially occurring tensions and biases between one or more (sub-)groups [32]. These inter-group biases should be prevented, for instance through an appropriate anonymization.

Step 3: Synopsis. The synopsis of data takes place both during and after the conduction of focus group sessions, representing an iterative data analysis. The purpose of this step is to compare spawning data with previous insights and re-frame the theoretical alignment of upcoming sessions accordingly. Throughout the iterative data synopsis, commonalities and conflicts within and between groups can be identified. This constant comparison leads to a recoding of the initial data set and the formation as well as refinement of subcategories [39]. As a result, the initial coding theme is (a) adjusted according to new insights and/or (b) complemented by new superordinate themes and associated subthemes.

Step 4: Design Extraction. Representing the first step of the deductive phase, the design extraction step seeks to identify and derive appropriate design principles based on the categorization scheme. Here, the researchers scan the data from a pragmatic (i.e. concrete design features and technological requirements) and a theoretical (i.e. related constructs and variables informing a kernel theory) perspective. This leads to the deductive formulation of design principles (e.g. "Keep users informed about the data captured") and associated constructs (e.g. "system transparency") in consideration of the quality criteria mentioned above (e.g. ethics).

Step 5. Theory Construction. The last step of the theory construction procedure consists of two consecutive steps: (a) Theoretical integration: Originating in Grounded Theory research, theoretical integration seeks to relate empirical evidence, which has been unearthed through iterative data collection and analysis, to theories and related constructs to be found in literature within the respective research field [35]. This way, researchers are able to identify kernel theories and adjacent constructs that relate to the emerged categories and design principles.

(b) Model generation: The final step of the empirical theory building procedure is the construction itself. Identified design principles (i.e. design items) build the design variations. They manifest one or more independent variables. These variables as a part of the kernel theory are constructs found in literature, and/or emerged during iterative data analysis. The dependent variable(s) also originate from pre-existing theories or empirical evidence. After the theoretical model has been developed, it can be empirically evaluated, for instance, by implementing the design variations and testing them in an experiment. In doing so, specific 
design variations of an artefact are validated by validating the design theory, while the design theory is validated by validating the artefact designs.

\section{Exemplary Application}

\subsection{Case Description}

The case described to illustrate the proposed design theory construction process is framed by a funded research project. Referring to the prerequisites described in chapter 3.1 , the scope and goals of the project, the technological boundaries and usage scenarios, as well as the composition of the participating stakeholders need to be depicted. The project case covers the development of a technical, mobile demonstrator, which is intended to support physiotherapeutic treatments following the so-called "Vojta" approach. The goal of the Vojta treatment is to make essential movement patterns available to the patient who suffers from an impairment of the central nervous system. The practitioner applies pressure to certain zones on the body of the patient, followed by automated physiological movements (e.g. arm movement) and other reactions (e.g. heart rate).

With regard to possible areas of system application, three major usage scenarios involving the system under development have been defined in an early stage of the project: (1) Since in many cases the Vojta treatment is performed by caregivers on the patients, the system is able to assist them with specific feedback (e.g. visual or auditory), guiding them during the session. On the upside, this could alleviate the necessity for clinical visits. (2) The system is collecting rich data during the treatments, capturing the quantity and quality of each session. This allows the physician in charge to control and, thus, improve the therapy progress, ultimately promising better health-related results. In addition, the control function is able to benefit the patients' compliance. (3) Due to its ability to guide users during their treatment sessions, the system can be applied as a teaching tool for apprentices. The technological boundaries are set up by diverse software and hardware components. In order to provide a proper measurement of body movements and relevant bio signals, the system involves depth cameras, a variety of body-worn sensors, and pressure plates the patient can be placed on. As the final prerequisite, the implementation of the described project calls for several disciplines, each exhibiting needed expertise and in-depth knowledge with regard to IT design and development, medicine and physiotherapy, as well as computer and social sciences. Hence, a multi-disciplinary group of 19 stakeholders from different domains has been acquired, covering the mentioned demands while holding individual values, judgments and opinions.

\subsection{Procedure and Findings}

Step 1: Ideation. The initial ideation took place in form of a "full group" [10] workshop involving all of the 19 participants from the diverse disciplines mentioned above, involving a moderator and a recording clerk. The session took 120 minutes in total. After a brief presentation of the usage scenarios by the moderator in order to establish a common understanding of the system, each participant was asked to write down system requirements and designrelevant issues that come to mind with regard to the system's future application and its impacts on the users, society, as well as the institutional frame. The individual results were presented in plenary. Simultaneously, the moderator loosely categorized and continuously re-arranged the mentioned topics, visualizing them on a whiteboard. As a result, four main categories emerged as an initial data set. The categories, their definitions, and respective example codes are presented in Table 1.

Step 2: Focused Exploration. Following the initial workshop, three subsequent focus group sessions have been conducted. The first session $(n=7)$ incorporated experts from the IT sector, i.e. developers and consultants. A group of computer and social scientists took part in the second workshop $(n=6)$. The last session $(n=6)$ involved physicians and therapists from the medical sector. Representing a "mini group" design [10], each focus group session took 90 minutes. The involvement of a homogeneous group with a shared space of expertise and meaning allowed for deep investigations on the identified categories from specific perspectives. Following the iterative approach, the findings from each session informed the subsequent ones, which fostered a theoretically driven data emergence. This allowed the focus group executives to address diverse or even antagonizing statements and opinions, leading to rich and multifaceted insights.

Step 3: Synopsis. The activity of constant data integration and comparison took place between each session and during the retrospective data analysis. By doing so, a comprehensive categorization scheme emerged, covering the initial main categories and the respective subcategories. For the sake of demonstrating the methodology presented in this paper, only one subcategory per main category is described below. The specific subcategories are selected based on (1) their substance regarding concrete design requirements and (2) their ability to inform the design items of an ISDT.

Individualized guidance (Autonomy): The system can offer different forms of guidance in accordance 
with the user's will to perform treatments autonomously. Low levels of guidance, thus, would allow individual approaches, such as therapeutic handles that work best for this specific user.

Forms of feedback (Competence): Different forms of feedback (e.g. audio/video signal, haptic feedback) in reaction to the user's operations are able to foster competence and confidence. Especially subliminal forms (e.g. vibration) can lessen distraction of the user, leading to a more focused treatment potentially increasing learning effects and individual competence.

Heterogeneity of data (Diversity): By collecting a vast set of different data and not relying on single measurements, the system becomes applicable by a wider population. For instance, the physiological constitution of the patient (e.g. disembodiment) does not lower the therapy's effectiveness.

Visualization (Privacy): The data captured by the system can be visualized and stored in different ways, for instance, using a generic, humanoid model or real video footage. Hence, depending on the user's privacy preferences, sensible data (e.g. face, genital area) can be disguised without losing too much information.

Table 1. Initial categorization scheme.

\begin{tabular}{|l|l|}
\hline Category and Definition & Example codes \\
\hline $\begin{array}{l}\text { Autonomy: The degree to } \\
\text { which the user is able to } \\
\text { autonomously control the } \\
\text { system. }\end{array}$ & $\begin{array}{l}\text { Increasing dependence on } \\
\text { the system; Diffusion of } \\
\text { responsibility in case of } \\
\text { treatment errors }\end{array}$ \\
\hline $\begin{array}{l}\text { Competence: The degree } \\
\text { to which the system } \\
\text { preserves or increases } \\
\text { individual skills. }\end{array}$ & $\begin{array}{l}\text { Handling of system errors } \\
\text { by users; False system } \\
\text { guidance leading to } \\
\text { treatment errors }\end{array}$ \\
\hline $\begin{array}{l}\text { Diversity: The degree to } \\
\text { which the system is } \\
\text { applicable by } \\
\text { heterogeneous user } \\
\text { groups (age, culture, etc.). }\end{array}$ & $\begin{array}{l}\text { Discrimination through } \\
\text { objectification; } \\
\text { Reasonableness of system } \\
\text { design; Cultural } \\
\text { backgrounds }\end{array}$ \\
\hline $\begin{array}{l}\text { Privacy: The degree to } \\
\text { which the system ensures } \\
\text { privacy and data security. }\end{array}$ & $\begin{array}{l}\text { Concerns of caregivers; } \\
\text { Confidentiality of sensitive } \\
\text { data; Fear of data misuse }\end{array}$ \\
\hline
\end{tabular}

Step 4: Design Extraction. For the sake of simplicity and illustration, two of the subcategories described above are consulted for the design extraction and subsequent theory construction, without denying the other categories' ability to inform further studies and research agendas. Looking at the autonomy and competence categories, the tentative implementation and testing of varying individualization levels of guidance paired with different forms of feedback appears especially fruitful. The feedback, in this case, is provided in reaction to events during the treatment sessions, for example, in order to indicate a false or correct movement and suggest corrective or reinforcing measures accordingly. These measures refer to the system guidance during a session and, thus, can be termed as feedback content, forming a second design principle besides different visualization forms. With regard to the quality criteria of design features described in chapter 2.2, this subcategory exhibits high degrees of feasibility (i.e. easy and economic implementation), acceptability (i.e. low level of interference with norms and values), and scientific suitability. Regarding the design feature's suitability, the context of the system, i.e. support of physiotherapeutic treatments, implies enhanced effects regarding the users' behavior, reactions, and attitudes towards the system. Table 2 contains the resulting exemplary design principles.

Table 2. Exemplary design principles.

\begin{tabular}{|c|c|}
\hline Subcategory & Design principles \\
\hline \multirow{3}{*}{$\begin{array}{l}\text { Individualized } \\
\text { guidance } \\
\text { (Autonomy) }\end{array}$} & No guidance (system gives no advice) \\
\hline & $\begin{array}{l}\text { Standardized guidance (advice based on } \\
\text { standard treatment procedures) }\end{array}$ \\
\hline & $\begin{array}{l}\text { Individualized guidance (advice based } \\
\text { on individual patient and previous } \\
\text { sessions) }\end{array}$ \\
\hline \multirow{3}{*}{$\begin{array}{l}\text { Forms of } \\
\text { feedback } \\
\text { (Competence) }\end{array}$} & $\begin{array}{l}\text { Visual signal (e.g. LED light or screen } \\
\text { notification) }\end{array}$ \\
\hline & $\begin{array}{l}\text { Auditory signal (e.g. sound signal or } \\
\text { human voice) }\end{array}$ \\
\hline & $\begin{array}{l}\text { Haptic signal (e.g. vibration of a body- } \\
\text { worn device) }\end{array}$ \\
\hline
\end{tabular}

Since the therapeutic treatments are of a haptic nature and require manual operations (e.g. applying pressure to the patient's body), feedback in form of vibration appears to have significant effects on the way people apply the treatment. The comparison with other forms of feedback such as light or voice signals in combination with alternating levels of individualized guidance suggests meaningful experimental results.

Step 5: Theory Construction. At this point, several findings emerged that constitute potential components of the ISDT under construction. Firstly, autonomy and competence represent theoretical and normative constructs that have already been subject to numerous studies in IS research and other disciplines, thus opening up a wide field of theoretical anchor points. Secondly, the defined design principles provide the design items of an ISDT. 
(a) Theoretical integration: Looking at research studies on competence and the establishment of medical skills (e.g. the proper execution of a therapy session by a novice or layperson), the trend of using technological devices such as simulators within medical education becomes evident. Over time, the reduction of medical errors in order to increase patient safety has become more important. Additionally, today's outcome-based education styles call for new ways of competence assessment, which technologies are able to deliver [31]. Medical simulators allow novices to practice and master certain techniques and procedures as well as ways to assess different kinds of competence, following the framework for clinical assessment [23]. With regard to learning and the acquisition of skills and knowledge, recent studies payed much attention on how to design multimedia learning material and the way it is presented to the learner in order to increase learning performance and outcomes (see for instance [20]). A promising theoretical concept that has been utilized throughout studies is Media Richness Theory (MRT), originating in organizational learning [4]. The theory "makes predictions about behavior and outcomes in connection with various communication media", classifying different media "according to their degree of richness" [18] (p. 11). MRT proposes that the provision of multiple communication channels (here: between system and user) will lead to choosing the most suitable channel available when it comes to solving tasks. On the opposite, it is stated that the constraint of media to choose from will lead to quality losses of task outcomes [18].

(b) Model generation: Within the context of the case study, the induced design principles inform different levels of media richness, implying a high suitability of MRT as a kernel theory. As a dependent variable, learning outcome highly suits the therapeutic setting and the presented usage scenarios, while being perceived as a normative and desirable effect.

Figure 2 illustrates the resulting explanatory ISDT, incorporating the identified design principles operationalizing two independent variables derived from empirical data and associated literature. The measurement model exemplary encompasses generic measurements covering objective (which need to be adapted to the individual context) and subjective measures for learning performance and outcome. The deployment of a suitable measurement model, though, is a task within the actual experimental theory-testing set-up. Based on the model developed, nine different design variations of the artefact can be implemented and evaluated. Taking the results into account, the "optimal" design variation can improve the artefact and form the basis for the next design iteration.

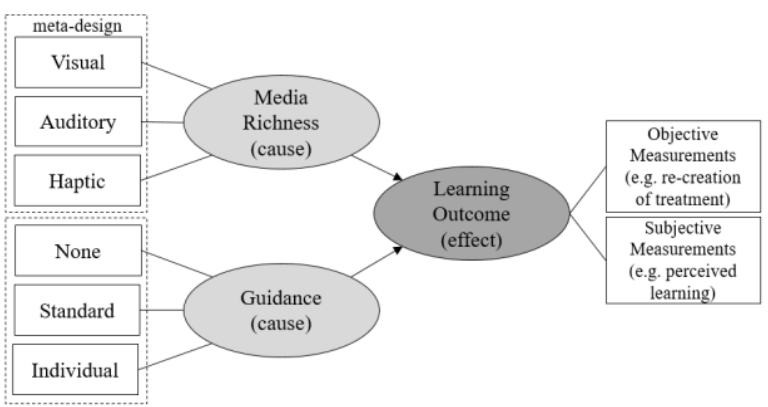

Figure 2. Resulting exemplary design theory.

\section{Conclusion}

Theoretical and practical implications. The initial step towards a framework, which allows for the inclusion of explorative, qualitative research methods to enrich the development process of an ISDT with empirical data presented in this paper exhibits several contributions to theory and practice. It contributes to a dual perspective on design theory and artefact design, in which theory and artefact evaluation go hand in hand. From a theoretical perspective, the paper contributes to the body of knowledge regarding design theorizing in DSR. The presented approach enables researchers to go beyond intuition and literature when it comes to develop new design theories. An empirical approach to set up design principles and associated constructs is able to improve the different design variations within an ISDT from multiple perspectives (e.g. societal, technological, and economical). The method is highly suitable within situative and specific contexts (e.g. joint research projects), satisfying particular requirements while addressing occurring and otherwise overlooked issues. When it comes to analyzing data and deriving design features and constructs, the resulting ISDTs have an immediate impact on the respective bodies of knowledge, informing further studies and illuminating effects IT artefacts exert on institutions and users. By empirically founding the design features within a design theory and testing them in an experimental setting, identified cause-effect relations and associated designs exhibit greater relevance for the implementation of the IT artefact. The practical advice an ISDT gives on which design features perform "best" regarding theoretical constructs is rooted in context-bound evidence and expertise, possibly leading to an advantageous IT deployment and adoption. Empirically constructed design theories inform feasible, desirable, and effective technology designs, easing operative decision making.

Methodological limitations. The methodological approach presented in this paper exhibits certain limitations. A major one is constituted by the data collection method itself, i.e. the focus group approach. 
Researchers have pointed out several issues when it comes to conducting and analyzing focus group sessions. On the one hand, partial or full conformity might occur during sessions based on the opinions, priorities, and views of dominant participants [32]. The resulting formation of sub-groups and related group dynamics can, thus, suppress individual views, the voice of minorities, as well as controversial opinions [24]. This might get reinforced with regard to normative discourses during sessions, which are prone to annihilate views that are contrary to perceived norms [32]. On the other hand, the trustworthiness, reliability and, thus, generalizability and external validity of findings might appear questionable, for instance due to idiosyncratic, off-the-cuff statements $[7,16]$. These limitations and issues require specific handling by the researcher(s), both from a pragmatic (i.e. moderator behavior during the session) and an analytic (i.e. interpreting the data) perspective [32].

Outlook. The paper at hand opens up several promising research opportunities and calls for supplementary method elaboration in order to achieve a methodological framework based on scientific rigor. Firstly, a further development of the presented approach utilizing complementary methods and data sources appears fruitful. Through a rigorous comparison of different methodological approaches and their suitability for inducing a theoretical baseline with regard to a technology design, the case of empirical design theory construction can be enriched, antagonizing some of the limitations of a pure focus group approach and, thus, profiting from inter-method synergies. Secondly, the triangulation of emerging empirical evidence with other data such as adjacent use cases seems promising. For instance, objective usage data derived from log-files or large-scale survey results capturing a variety of opinions and requirements are inclined to deliver more robust findings, allowing accurate and viable design theories to emerge. Thirdly, further method application in other projects enables its advancement and refinement, since underlying common goals of project members might evoke biases.

Acknowledgements. This article was supported by the SenseVojta project granted by the German Federal Ministry of Education and Research (BMBF) (No. 13GW0166E).

\section{References}

[1] Aggarwal, D., W. Zhang, T. Hoang, B. Ploderer, F. Vetere, and M. Bradford, "SoPhy: A Wearable Technology for Lower Limb Assessment in Video Consultations of Physiotherapy", Proceedings of the 2017 CHI Conference on Human Factors in Computing Systems, ACM Press (2017), 3916-3928.
[2] Baskerville, R., and J. Pries-Heje, "Explanatory design theory", Business \& Information Systems Engineering 2(5), 2010, pp. 271-282.

[3] Bybee, M., "Abductive inferences and the structure of scientific knowledge", Argumentation 10(1), 1996, pp. 2546.

[4] Daft, R.L., and R.H. Lengel, "Organizational information requirements, media richness and structural design", Management science 32(5), 1986, pp. 554-571.

[5] De Jouvenel, H., "A Brief Methodological Guide to Scenario Building", Technological Forecasting and Social Change 65(1), 2000, pp. 37-48.

[6] Dew, N., "Abduction: a pre-condition for the intelligent design of strategy", Journal of Business Strategy 28(4), 2007, pp. 38-45.

[7] Eisenhardt, K.M., and M.E. Graebner, "Theory building from cases: Opportunities and challenges", Academy of management journal 50(1), 2007, pp. 25-32.

[8] Eysenbach, G., "Recent advances: Consumer health informatics", BMJ: British Medical Journal 320(7251), 2000, pp. 1713.

[9] Friedman, B., P.H. Kahn Jr, A. Borning, and A. Huldtgren, "Value sensitive design and information systems", In Early engagement and new technologies: Opening up the laboratory. Springer, Dordrecht, 2013, 5595.

[10] Greenbaum, T.L., The handbook for focus group research, Sage, 1998.

[11] Gregor, S., "Building theory in the sciences of the artificial", ACM (2009), 4.

[12] Gregor, S., and D. Jones, "The anatomy of a design theory", Journal of the Association for Information Systems 8(5), 2007, pp. 312.

[13] Gregory, R.W., and J. Muntermann, "Research NoteHeuristic theorizing: Proactively generating design theories", Information Systems Research 25(3), 2014, pp. 639-653.

[14] Hevner, A., S.T. March, J. Park, and S. Ram, "Design science in information systems research", MIS quarterly 28(1), 2004, pp. 75-105.

[15] Judi, H.M., A. Razak, N. Sha'ari, and H. Mohamed, "Feasibility and critical success factors in implementing telemedicine", Information Technology Journal 8(3), 2009, pp. 326-332.

[16] Kidd, P.S., and M.B. Parshall, "Getting the focus and the group: enhancing analytical rigor in focus group 
research", Qualitative health research 10(3), 2000, pp. 293308.

[17] Kitzinger, J., "Qualitative research. Introducing focus groups.”, BMJ: British medical journal 311(7000), 1995, pp. 299.

[18] Kock, N., "Information systems theorizing based on evolutionary psychology: an interdisciplinary review and theory integration framework", Mis Quarterly, 2009, pp. 395-418.

[19] Kuechler, W., and V. Vaishnavi, “A framework for theory development in design science research: multiple perspectives", Journal of the Association for Information systems 13(6), 2012, pp. 395.

[20] Liu, S.-H., H.-L. Liao, and J.A. Pratt, "Impact of media richness and flow on e-learning technology acceptance", Computers \& Education 52(3), 2009, pp. 599-607.

[21] March, S.T., and G.F. Smith, "Design and natural science research on information technology", Decision support systems 15(4), 1995, pp. 251-266.

[22] Marshall, M.N., "Sampling for qualitative research", Family practice 13(6), 1996, pp. 522-526.

[23] Miller, G.E., "The assessment of clinical skills/competence/performance.”, Academic medicine 65(9), 1990, pp. S63-7.

[24] Morgan, D., Focus Groups as Qualitative Research, SAGE Publications, Inc., 2455 Teller Road, Thousand Oaks California 91320 United States of America, 1997.

[25] Niehaves, B., and K. Ortbach, "The inner and the outer model in explanatory design theory: the case of designing electronic feedback systems", European Journal of Information Systems 25(4), 2016, pp. 303-316.

[26] O'hEocha, C., X. Wang, and K. Conboy, "The use of focus groups in complex and pressurised IS studies and evaluation using Klein \& Myers principles for interpretive research: Evaluate an applied focus group approach using Klein \& Myers principles", Information Systems Journal 22(3), 2012, pp. 235-256.

[27] Owen, R., J. Stilgoe, P. Macnaghten, M. Gorman, E. Fisher, and D. Guston, "A framework for responsible innovation", Responsible innovation: managing the responsible emergence of science and innovation in society, 2013, pp. 27-50.

[28] Peffers, K., T. Tuunanen, M.A. Rothenberger, and S. Chatterjee, "A design science research methodology for information systems research", Journal of management information systems 24(3), 2007, pp. 45-77.
[29] Pot, A.M., B.M. Willemse, and S. Horjus, "A pilot study on the use of tracking technology: Feasibility, acceptability, and benefits for people in early stages of dementia and their informal caregivers", Aging \& Mental Health 16(1), 2012, pp. $127-134$.

[30] Powell, R.A., and H.M. Single, "Focus groups", International journal for quality in health care 8(5), 1996, pp. 499-504.

[31] Scalese, R.J., V.T. Obeso, and S.B. Issenberg, "Simulation Technology for Skills Training and Competency Assessment in Medical Education", Journal of General Internal Medicine 23(S1), 2008, pp. 46-49.

[32] Smithson, J., "Using and analysing focus groups: limitations and possibilities", International journal of social research methodology 3(2), 2000, pp. 103-119.

[33] Stahl, B.C., M.C. Tremblay, and C.M. LeRouge, "Focus groups and critical social IS research: how the choice of method can promote emancipation of respondents and researchers", European Journal of Information Systems 20(4), 2011, pp. 378-394.

[34] Stewart, D.W., and P.N. Shamdasani, Focus groups: Theory and practice, Sage publications, 2014.

[35] Urquhart, C., H. Lehmann, and M.D. Myers, "Putting the 'theory' back into grounded theory: guidelines for grounded theory studies in information systems: Guidelines for grounded theory studies in information systems", Information Systems Journal 20(4), 2009, pp. 357-381.

[36] Venkatesh, V., M.G. Morris, G.B. Davis, and F.D. Davis, "User acceptance of information technology: Toward a unified view", MIS quarterly, 2003, pp. 425-478.

[37] Walls, J.G., G.R. Widmeyer, and O.A. El Sawy, "Building an information system design theory for vigilant EIS”, Information systems research 3(1), 1992, pp. 36-59.

[38] Wang, W., Y. Zhao, L. Qiu, and Y. Zhu, "Effects of Emoticons on the Acceptance of Negative Feedback in Computer-Mediated Communication", Journal of the Association for Information Systems 15(8), 2014, pp. 454483.

[39] Wiesche, M., M.C. Jurisch, P.W. Yetton, and H. Krcmar, "Grounded Theory Methodology in Information Systems Research”, MIS Quarterly 41(3), 2017, pp. 685-701.

[40] Zhu, L., I. Benbasat, and Z. Jiang, "Let's Shop Online Together: An Empirical Investigation of Collaborative Online Shopping Support”, Information Systems Research 21(4), 2010, pp. 872-891. 